\title{
Diseño del programa de apoyo psicoeducativo para promover la resiliencia parental "Supéra- te. ¡No tires la toalla!”
}

\section{María Cantero-García ${ }^{1}$, Helena Garrido-Hernansaiz ${ }^{2}$ y Jesús Alonso-Tapia ${ }^{3}$}

${ }^{1}$ Facultad de Ciencias Biomédicas y de la Salud. Universidad Europea de Madrid \& Área de Salud, Universidad Internacional de Valencia. Valencia ${ }^{2}$ Centro Universitario Cardenal Cisneros. Universidad de Álcala de Henares. Madrid.

${ }^{3}$ Universidad Autónoma de Madrid. Facultad de Psicología. Madrid

\section{España}

Correspondencia: María Cantero García. Universidad Internacional de Valencia C/ Pintor Sorolla, 21 CP: 46002 (Valencia) E-mail: maria.canterogar@gmail.com 


\section{Resumen}

Introducción. Los problemas de conducta de niños y/o adolescentes constituyen una de las preocupaciones de las familias en la sociedad actual. Ante esta problemática, hay familias que son capaces de afrontar estos problemas y salir adelante, mientras otras se ven desbordadas, sin saber cómo actuar. Esta diferencia puede relacionarse con un uso inadecuado de estrategias de autorregulación emocional, estrategias de afrontamiento eficaces y niveles bajos de resiliencia.

Método. Para ayudar a superar tales carencias se diseñó un programa orientado a mejorar las habilidades de las familias para el manejo de los problemas de comportamiento de sus hijos, así como, las estrategias de afrontamiento y regulación emocional de los padres (se utilizaron técnicas cognitivas conductuales, así como, técnicas de aceptación y compromiso).

Resultados. Los resultados revelaron que el programa de intervención provocó una disminución significativa en los niveles de ansiedad, depresión, así como una mejora en el clima familiar percibido. El objetivo del presente estudio fue evaluar la efectividad del mismo. Participaron 61 padres, 41 en el grupo experimental y 20 en el de control. Para determinar la efectividad del programa se evaluaron los cambios: a) en los padres: en conocimiento de las estrategias de actuación, en el clima familiar percibido, y los niveles de resiliencia, ansiedad/estrés y depresión; b) en los hijos: percepción del clima familiar.

Discusión y conclusiones. Los resultados revelaron que el programa de intervención provocó una disminución significativa en los niveles de ansiedad, depresión, así como una mejora en el clima familiar percibido.

Palabras Clave: estrategias de afrontamiento, resiliencia, problemas de comportamiento, intervención 


\begin{abstract}
Introduction: Children's and adolescents' behavioral problems are one of the main concerns in today's society families. Confronted with these problems, there are families that can very well cope with them and get ahead, whereas other families are overwhelmed without knowing how to act. This difference may be related the non appropriate use of emotional selfregulation strategies or confrontation strategies and also low levels of resilience.

Method: In order to overcome such issues, we designed a program aiming to improve families' capabilities to deal with the mentioned behavioral problems, as well as confrontation strategies and parents' levels of emotional regulation (we used cognitive behavioral techniques as well as techniques of acceptance and commitment).

Results: The main objective of this study was to evaluate the effectiveness of this program. Sixty-one parents took part in our study, forty-one in the experimental group and twenty in the control group. To determine our program effectiveness, we evaluate changes: a) concerning parents: knowledge of strategies to cope with problems, perceived family climate, and levels of resilience, anxiety/stress and depression, b) concerning children: perceived family climate.
\end{abstract}

Discussion or Conclusion: Results suggest that our intervention produced a significant reduction in the levels of anxiety, depression as well as an improvement in the perceived family climate.

Keywords: coping strategies; coping assessment, resilience, behavior problems, intervention 


\section{Introducción}

Los problemas de conducta que muestran los hijos constituyen un importante estresor en el ámbito familiar (Herreros, Sánchez, Rubio y Gracia, 2004). Estos problemas y la forma de afrontarlos generan interferencias en el clima familiar, incrementando el estrés asociado a la crianza y reduciendo el bienestar emocional y los niveles de resiliencia parentales.

Una de las formas de intervención con mejores resultados para tratar los problemas de conducta son los Programas de Entrenamiento para Padres (Chorpita et al., 2011; Forehand, Jones y Parent, 2013; Morales Chainé, Martínez, Martín del Campo y Nieto, 2016). El principal objetivo de dichos programas es enseñar a los padres técnicas para la modificación de la conducta, tales como reforzar el buen comportamiento y otras estrategias no punitivas (Robles y Romero, 2011). Los distintos programas ponen su atención en enseñar estrategias como: establecer límites de forma adecuada, manejar los problemas de comportamiento mediante técnicas como el tiempo fuera. Entre ellos están: Triple-P (Sanders, Dadds y Turners, 2003), Incredible Year (Webster Stratom et al, 2003), Coping Power (Lochman y Wells, 2002; Lochman et al., 2007), EmPeCemos (Romero, Villar, Luengo, Gómez-Fraguela y Robles, 2009), Vivir la Adolescencia en Familia: Un programa de apoyo parental en la adolescencia (Rodríguez, Martín y Rodrigo, 2015). Más recientemente, otros programas están añadiendo otros componentes como el manejo de la ira, habilidades sociales, habilidades comunicativas y /o resolución de conflictos (Lozano y Valero, 2017).

Además, la mayoría de estos programas se han centrado en tratar los problemas de conducta desde el punto de vista patológico, olvidando en la mayoría de los casos trabajar las fortalezas y potencialidades de los padres de modo preventivo, como se indicaba en la Recomendación Rec (2006) 19 del Comité de Ministros a los Estados Miembros sobre políticas de apoyo al ejercicio positivo de la parentalidad (Consejo de Europa, 2006). Además, aún es notoria la falta de programas dirigidos a trabajar estrategias de afrontamiento y autorregulación emocional, así como estrategias de manejo de los problemas de conducta para que mejore el sentido de autoeficacia parental. Por este motivo, se hace necesario el diseño del programa de intervención psicoeducativa: ¡Supérate, no tires la toalla! 
La selección de las estrategias de afrontamiento a utilizar en el programa (pensamiento positivo, relajación, manejo de la culpa, detención de la rumiación y búsqueda de ayuda) se ve respaldada por estudios como los de Alonso-Tapia, Garrido-Hernansaiz, Rodríguez-Rey, Ruiz y Nieto (2017), que evidencian que trabajar este tipo de estrategias de afrontamiento contribuye a la mejora de los niveles de resiliencia y bienestar emocional. Además, en esta línea y siguiendo a autores como Azar y Cote (2002) se han trabajado competencias parentales (p.ej., control de impulsos, habilidades de autocontrol, solución de problemas interpersonales, empatía, reconocimiento emocional). Por otro lado, las estrategias relacionadas con la autoeficacia parental han sido validadas en los programas citados anteriormente.

El programa diseñado tiene como base la Recomendación Rec (2006)19 del Comité de Ministros a los Estados Miembros sobre políticas de apoyo al ejercicio positivo de la parentalidad (Consejo de Europa, 2006). Además, utiliza la metodología experiencial, que ha sido validada en programas de educación parental (Galbraith y Fouch, 2007; Martín, Máiquez, Rodrigo, Correa y Rodríguez, 2004; Rodrigo, Martín, Máiquez y Rodríguez, 2005). El sentido de la metodología experiencial implica seguir un proceso de reflexión y de análisis de las prácticas educativas, de las consecuencias de estas en la vida familiar o en el desarrollo de los hijos para propiciar finalmente la verbalización de los objetivos del proceso de cambio. La metodología experiencial, tal y como recoge Maiquez et al., (2000) genera la oportunidad de conocer una amplia gama de experiencias variadas, a través de las cuales se intenta promover cambios a nivel cognitivo, emocional y comportamental en las familias. Desde este modelo se apunta a la optimización de capacidades y no solo a la compensación de los déficits, partiendo de la idea que señala que la manera de actuar de los padres y madres en la crianza y educación tiene un rol central. De este modo, nos encontramos con la necesidad de elaborar actividades que fomenten la participación y el intercambio de experiencias entre los participantes (Rodrigo, Máiquez y Martín, 2015). A lo largo de las sesiones se utilizarán técnicas grupales propias de la metodología experiencial tales como la técnica de estudio de casos, la discusión dirigida, role-playing, las tareas para trabajar en casa y/o los compromisos. Además, se elaboran otro tipo de dinámicas y actividades para cada sesión en concreto. Todas estas actividades y técnicas están adaptadas a los objetivos de cada sesión.

Con esta metodología, el papel del facilitador es ayudar a construir de manera compartida el conocimiento en el grupo, partiendo de las experiencias previas de los participantes (Martínez-González, Rodríguez-Ruiz, Álvarez-Blanco y Becedóniz-Vázquez, 2016; Rodrigo, 
Máiquez y Martín, 2015), y para ello se deben tener en cuenta una serie de principios como: regular el clima emocional del grupo, construir el conocimiento como un miembro más, trabajar a partir de las necesidades, experiencias y preocupaciones de las familias, fomentar la negociación y búsqueda de consenso entre los miembros del grupo, apoyar la iniciativa personal y reforzar los sentimientos de pertenencia al grupo.

\section{Objetivos e hipótesis}

Teniendo como base de la intervención estas fortalezas y partiendo del análisis del entorno y la importancia que tiene trabajar las estrategias de afrontamiento y la autoeficacia de los padres, se ha desarrollado y puesto a prueba esta intervención, cuyos objetivos son investigar: a) si es posible enseñar a los padres estrategias de afrontamiento para el manejo del estrés generado por los problemas de conducta de sus hijos, y estrategias de manejo de estos problemas para que aumente su sentido de autoeficacia a la hora de enfrentarse con ellos; b) si dicha enseñanza influye en sus niveles de resiliencia, bienestar emocional y en el clima familiar; c) si el cambio producido en el clima familiar es percibido por los hijos; d) si las variables edad de los progenitores, edad de los niños, tipo de familia, etc. modulan la mayor o menor efectividad del programa.

La consecución de los objetivos señalados puede manifestarse mediante distintos indicadores, por lo que se evaluará la ocurrencia de cambios en las siguientes variables: 1) los conocimientos de los padres sobre las estrategias a utilizar para manejar los problemas de comportamiento de los hijos, 2) la mejora en el uso de las estrategias de afrontamiento y autorregulación emocional, 3) los cambios en la satisfacción vital y la salud mental de los padres (estrés, ansiedad y depresión), así como el incremento en su competencia parental, 4) La mejora en el clima familiar, percibido, no sólo por los padres sino también por los hijos, y 5) el grado de satisfacción con el programa. La hipótesis general es que los cambios en las variables dependientes se producirán en la dirección esperada y serán significativamente mayores en el grupo experimental que en el control. Además, esperamos que el estudio nos permita conocer los puntos fuertes y débiles de la intervención, de cara a su futura mejora. 


\section{Método}

\section{Participantes}

Participaron 61 padres de siete centros de educación secundaria de la Comunidad de Madrid, escogidos mediante un muestreo no probabilístico por conveniencia. De los siete centros, tres eran centros públicos, otros dos eran centros concertados y dos eran centros privados. Del total de inscritos, los pertenecientes a seis centros $(n=41)$ fueron asignados al grupo experimental y los pertenecientes al séptimo centro (público) $(n=20)$ fueron asignados al grupo control de lista de espera. Todos los participantes de ambos grupos completaron todas las evaluaciones.

De los 61 participantes, 47 son mujeres y 14 hombres, con edades comprendidas entre los 31 y los 54 años $(M=44,26$ años; $D T=4,64)$. En cuanto al nivel educativo, el 70,5\% tienen estudios universitarios, el 22,9\% tienen bachillerato o FP y el 6,6\% tienen educación primaria o secundaria. El 80\% de los participantes tiene un trabajo remunerado. El 88,5\% de participantes están casados/as, el 3,3\% están solteros/as y el 8,2\% están separados o divorciados. El número de hijos de los participantes oscila entre 1 y $3(M=2 ; D T=.48)$ con edades comprendidas entre los 9 y $\operatorname{los} 15$ años $(M=11,38 ; D T=1,64)$. El $44 \%$ son niñas y el $56 \%$ niños. Todos los y las participantes tienen nacionalidad española.

\section{Instrumentos}

\section{Materiales de intervención.}

Para facilitar el aprendizaje, el procedimiento utilizado en el diseño de este programa ha supuesto prestar atención a la estructura, duración y distribución de las sesiones, a la metodología, y a la dinámica de las mismas. El programa se lleva a cabo de manera grupal. La duración de cada sesión es de 90 minutos, y su distribución es semanal, dado que una semana parece un tiempo apropiado para que los padres y madres puedan reflexionar sobre lo trabajado en cada sesión e intentar poner en práctica las habilidades y estrategias propuestas, de modo que puedan probar su efectividad (Rodrigo, Máiquez y Martín, 2015). En cuanto a su estructura, cada sesión consta de las siguientes fases: 1) Entrada, saludo y bienvenida de los participantes. 2) Repaso de lo tratado en la sesión anterior, identificar las dificultades percibidas en la puesta en práctica. 3) Presentación de nuevos contenidos. 4) Introducción de las habilidades parentales asociadas a los contenidos de cada sesión. 5) Resumen de lo tratado. Entrega de la síntesis a recordar. 6) Despedida y cierre. 
Puesto que el primer objetivo es que los padres aprendan, en primer lugar, estrategias que les permitan manejar los problemas de conducta de sus hijos, se han seleccionado las siguientes estrategias de eficacia demostrada: uso del refuerzo, alternativas al castigo y estrategias para la resolución de conflictos en familia (Martínez-González, Rodríguez-Ruiz, Álvarez-Blanco, Becedóniz-Vázquez,2016; Ponzetti, 2016; Rodrigo, Máiquez y Martín, 2015).

Asimismo, puesto que el segundo objetivo es que los padres adquieran y/o mejoren sus estrategias de afrontamiento del estrés y autorregulación de sus emociones, de modo que se potencie su resiliencia, se han seleccionado para su inclusión en el programa las siguientes estrategias de afrontamiento: pensamiento positivo, relajación, manejo de la culpa, detención de la rumiación y búsqueda de ayuda, cuya efectividad ha sido justificada anteriormente. Estas estrategias se han trabajado en relación directa con los problemas de conducta de los hijos.

El entrenamiento de los dos tipos de estrategias enumeradas constituye el contenido del programa, que se ha organizado en 10 sesiones. Cada sesión (de la 1 a la 6) está dividida en dos partes, en la primera parte se trabajan las distintas técnicas que nos van a permitir mejorar la autoeficacia parental, así como mejorar el clima familiar y reducir los problemas de comportamiento de los hijos. La segunda parte de la sesión, (y de la sesión 7 a la 10), está destinada a trabajar las distintas estrategias de afrontamiento que contribuyen a la mejora de los niveles de resiliencia y del bienestar emocional parental. Además, consta con dos sesiones extras, una antes de la intervención y una después para la evaluación.

En primer lugar, se desarrolla una sesión cero o sesión inicial cuyo objetivo principal es promover la familiarización del facilitador con el grupo de participantes y viceversa, además de establecer las primeras normas del grupo (confidencialidad, respeto, etc.). También, se presenta a las familias los objetivos-contenidos del programa y se reflexiona brevemente sobre la importancia de trabajar los problemas de conducta. A continuación, describimos brevemente cada una de las sesiones.

Sesión 1.- El comportamiento de nuestros hijos. El objetivo de esta sesión es reflexionar sobre la importancia que tiene conocer el comportamiento de los hijos, así como las características propias de su etapa evolutiva. Se subraya la importancia de atender la conducta en términos específicos, evitando etiquetar al niño con atributos negativos (ej., "tienes la habita- 
ción bastante desordenada" en vez de "eres un desastre"). Además, a lo largo de esta sesión se pretende que los padres identifiquen la espiral de interacciones negativas que puede estar afectando a las relaciones con su hijo (y se insiste en la necesidad de prestar atención a las conductas positivas. A modo de conclusión, se discuten, modelan y practican formas adecuadas de elogio. Además, en la segunda parte de la sesión los padres comienzan a trabajar el autoconocimiento, el autoelogio y la importancia del autocuidado.

Sesión 2. - Nos ponemos en los zapatos de nuestros hijos. - Educar en y desde la asertividad y la empatía es uno de los procesos fundamentales implicados en el desarrollo de las relaciones familiares. A lo largo de esta sesión, se reflexiona acerca de las características que diferencian a una persona asertiva de una agresiva o pasiva, además, se sigue trabajando sobre el autoconocimiento parental, así como sobre la importancia que tiene ponernos en el lugar de los hijos para comprender mejor su forma de actuación.

Sesión 3. - Aprendemos a comunicarnos en familia. - Esta sesión se dedica a potenciar las habilidades de comunicación que son necesarias para un buen funcionamiento familiar. Estas estrategias serán la base y el apoyo de otras estrategias parentales que veremos sesiones posteriores. En la última parte de esta sesión se continúa trabajando el tema del autocuidado parental y la importancia de los valores.

Sesión 4. - Regulamos el comportamiento I. En esta sesión se trabaja la transmisión de órdenes eficaces, el establecimiento de límites razonables y predecibles e ignorar las conductas perturbadoras poco importantes. Los padres aprenden a formular adecuadamente sus órdenes y peticiones, evitando el exceso de instrucciones innecesarias, en cadena o formuladas en forma de pregunta. Se presenta la técnica de "ignorar" como técnica para responder a la mala conducta. En la segunda parte de esta sesión se trabaja el tema de la culpa.

Sesión 5. - Regulamos el comportamiento II. Esta sesión se dedica al uso de las consecuencias negativas ante el mal comportamiento. Los padres aprenden reconocer las características que definen unas consecuencias eficaces (inmediatez, consistencia, proporcionalidad) y aprenden, principalmente a establecer y poner en práctica un tipo particular de consecuencias: las consecuencias lógicas y las naturales. Además, aprenden a establecer un sistema de consecuencias razonables, predecibles y adecuadas a la edad y capacidad del niño (retirada de privilegios, trabajos extra). La "técnica tiempo fuera" se presenta como una alternativa al castigo 
físico. Sin embargo, se enfatiza que el uso de consecuencias negativas deberá reducirse al mínimo, en favor de las formas de interacción basadas en el reforzamiento de la conducta. Esta sesión aborda las expectativas de los padres sobre los comportamientos a promover en sus hijos y profundiza en el uso adecuado de refuerzos tangibles como complemento al elogio. Los padres diseñan, un sistema para esos comportamientos que deseen instaurar o consolidar en sus hijos. En la segunda parte de esta sesión se aborda el tema del perdón.

Sesión 6. - Sin estrés estamos mejor. Acercarse a las situaciones de estrés y sus consecuencias para la dinámica familiar, así como trabajar técnicas para su manejo son algunos de los aspectos que se trabajarán en esta sesión. A lo largo de esta sesión conocerán distintas técnicas para afrontar de forma más saludable el estrés causado por los problemas de comportamiento de sus hijos. En la segunda parte de esta sesión se trabaja sobre la importancia del autocontrol y la autorregulación emocional.

Sesión 7. - Pensamos y sentimos en positivo. A lo largo de esta sesión se trabaja en primer lugar sobre la resolución de conflictos en familia. La segunda parte trabaja la importancia de cambiar los pensamientos negativos por otros más optimistas, la importancia de cambiar las ideas irracionales y las autoinstrucciones desadaptativas. De forma más operante se trabaja el tema de la aceptación.

Sesión 8. - Superamos las dificultades sin tirar la toalla. A lo largo de la sesión se reflexiona con los participantes sobre el concepto de resiliencia y la importancia que tiene tener presente los aspectos tratados en las distintas sesiones (aceptación, no rumiación, relajación, pensamiento positivo, autoculpabilización, perdón), así como las distintas estrategias de afrontamiento centradas en el problema (reintentar, pedir ayuda, buscar soluciones) y estilos de afrontamiento para la mejora de su bienestar emocional y familiar.

Sesión 9. - Con H de Humor y A de Amor. En esta penúltima sesión, se trabaja el humor como estrategia de afrontamiento para reducir la tensión en los momentos de estrés. Se trata de concienciar a las familias de la importancia de tomarse las cosas con sentido del humor, así como de sus beneficios.

Sesión 10. - Sesión final. La sesión final se dedica a recapitular las técnicas aprendidas, tanto para el manejo de los comportamientos de los hijos como para la mejora del bienes- 
tar emocional de los padres. Una vez más, resaltamos la importancia del bienestar parental para poder actuar de forma eficaz. Finalmente, se prepara a los padres para el afrontamiento de los problemas de conducta que puedan surgir una vez que finalice el programa, se presentarán distintas estrategias para prevenir las recaídas y se potenciará la autoeficacia para enfrentarse a los retos que plantea la educación de sus hijos. En esta última sesión se efectúa también la evaluación final del programa.

\section{Materiales de evaluación.}

La evaluación del programa se apoya en estudios como el Grant et al. (2018) y la propuesta de Alonso-Tapia (2012, p. 341), cuyo esquema se presenta en la Figura 1.

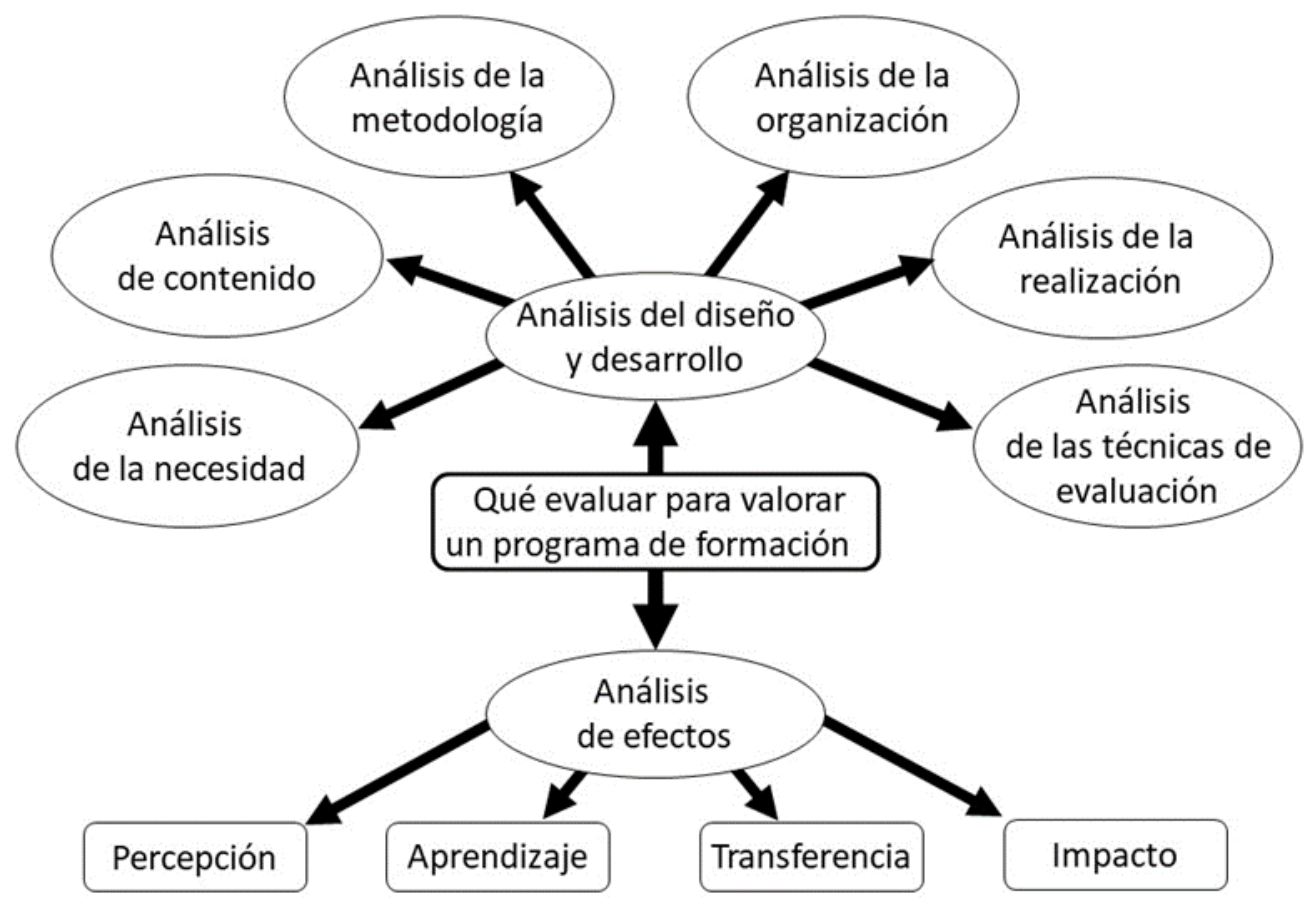

Figura 1. Elementos a considerar en la valoración de programas (Alonso-Tapia, 2012)

Dicha propuesta recoge los elementos necesarios a considerar en un programa de intervención (Flay et al., 2005; Gottfredson et al., 2015): 1) Evaluación del aprendizaje: “evalúa el aprendizaje logrado por los destinatarios en relación con los objetivos conseguidos". 2) Evaluación de la transferencia: “evalúa si los participantes utilizan esos conocimientos en el contexto apropiado". 3) Evaluación del impacto: "evalúa si un programa puede tener impacto en otras características de los participantes diferentes de las entrenadas o en otras personas", en este caso, si los efectos son percibidos por los hijos. 4) Evaluación de la percepción de la 
calidad: "evalúa la percepción que los participantes tienen del programa en cuestiones relacionadas con la satisfacción, la organización, etc.". Sobre la base de esta propuesta se han seleccionado las variables a medir y los instrumentos a utilizar para medirlas, incluidos en la Tabla 1 junto con la descripción y la fiabilidad de las escalas que los integran.

Tabla 1. Instrumentos utilizados para la evaluación de la intervención.

\begin{tabular}{|c|c|}
\hline Instrumentos & Propiedades psicométricas \\
\hline \multicolumn{2}{|r|}{ EVALUACIÓN DE APRENDIZAJE } \\
\hline $\begin{array}{l}\text { Cuestionario de Conocimien- } \\
\text { tos previos sobre gestión del } \\
\text { comportamiento y afronta- } \\
\text { miento del estrés. } \\
\text { (Cantero-García y Alonso- } \\
\text { Tapia, 2018c) }\end{array}$ & $\begin{array}{l}\text { Consta de } 22 \text { ítems con tres opciones de respuesta. En la tabla } 2 \text { se } \\
\text { presenta un ejemplo de sus ítems. Ha sido desarrollado para este estu- } \\
\text { dio. }\end{array}$ \\
\hline \multicolumn{2}{|r|}{ EVALUACIÓN DE TRANSFERENCIA } \\
\hline $\begin{array}{l}\text { Cuestionario de clima familiar, } \\
\text { perspectiva de los padres } \\
\text { (Cantero-García y Alonso- } \\
\text { Tapia, 2017a) }\end{array}$ & $\begin{array}{l}\text { Incluye } 20 \text { ítems formulados de forma positiva y negativa, agrupados } \\
\text { en cuatro subescalas y una general. Hacen referencia a cuatro tipos de } \\
\text { reacciones castigo vs razonamiento, estrés y actitud positiva. Los índi- } \\
\text { ces de fiabilidad son: escala general de Clima Familiar ( } \omega: .94) \text { razo- } \\
\text { namiento vs castigo ( } \omega: .84) \text {; paciencia ( } \omega: .81) \text {; estrés }(\omega: .88) \text { y acti- } \\
\text { tud positiva ( } \omega: .87) \text {. Los ítems se contestan en una escala Likert de } \\
\text { cinco puntos siendo } 1 \text { (totalmente en desacuerdo) y } 5 \text { (totalmente de } \\
\text { acuerdo). }\end{array}$ \\
\hline $\begin{array}{l}\text { Cuestionario de Estrategias de } \\
\text { Afrontamiento frente a los } \\
\text { problemas de conducta de los } \\
\text { hijos (CAF). } \\
\text { (Cantero-García y Alonso- } \\
\text { Tapia, 2018a) }\end{array}$ & $\begin{array}{l}\text { El instrumento incluye } 36 \text { ítems agrupados en } 12 \text { categorías básicas - } \\
\text { tres por categoría-, agrupadas a su vez en tres categorías que recogen } \\
\text { los estilos de afrontamiento. Los ítems formulados tanto de forma posi- } \\
\text { tiva como negativa se responden en una escala Likert señalando el } \\
\text { grado de acuerdo desde } 1 \text { (totalmente en desacuerdo) a } 5 \text { (totalmente } \\
\text { de acuerdo). La fiabilidad de las escalas es buena oscila entre } .64 \text { y .91 }\end{array}$ \\
\hline \multicolumn{2}{|r|}{ EVALUACIÓN DE IMPACTO } \\
\hline $\begin{array}{l}\text { Cuestionario de percepción de } \\
\text { los problemas de conducta de } \\
\text { los hijos. } \\
\text { (Cantero- García y Alonso- } \\
\text { Tapia, 2017b) }\end{array}$ & $\begin{array}{l}\text { Incluye } 10 \text { ítems que hacen referencia a los distintos problemas de } \\
\text { conducta que presentan los hijos. Los ítems se miden en escalas Likert } \\
\text { señalando el grado en el que se manifiestan siendo } 1 \text { (muy poco) y } 5 \\
\text { (mucho). }\end{array}$ \\
\hline
\end{tabular}


Tabla 1 (Continua). Instrumentos utilizados para la evaluación de la intervención.

\begin{tabular}{|c|c|}
\hline Instrumentos & Propiedades psicométricas \\
\hline & EVALUACIÓN DE IMPACTO \\
\hline $\begin{array}{l}\text { Cuestionario de clima familiar, } \\
\text { perspectiva de los hijos. (Cante- } \\
\text { ro- García y Alonso-Tapia, } \\
2017 \text { c) }\end{array}$ & $\begin{array}{l}\text { Evalúa la percepción de los hijos sobre la forma de actuar de los padres ante } \\
\text { los problemas de conducta. Incluye } 20 \text { ítems que hacen referencia a cuatro } \\
\text { estrategias: actitud positiva, razonamiento vs castigo, paciencia y estrés. Los } \\
\text { ítems formulados tanto de forma positiva como de manera negativa se res- } \\
\text { ponden señalando el grado de acuerdo en una escala Likert de cinco puntos } \\
\text { (siendo } 1 \text { totalmente en desacuerdo y } 5 \text { totalmente de acuerdo). La consisten- } \\
\text { cia interna de la escala completa, evaluada mediante el índice } \alpha \text { de Cronbach, } \\
\text { fue de } .84 \text {. Las escalas individuales obtuvieron los siguientes índices de fiabi- } \\
\text { lidad: castigo vs razonamiento, } \alpha: .55 \text {; paciencia, } \alpha: .67 \text {; estrés, } \alpha: .77 \text { y actitud } \\
\text { positiva, } \alpha=.61 \text {. En la escala criterio, el } \alpha \text { de Cronbach fue de } .74 \text {, lo que nos } \\
\text { indica que es aceptable. }\end{array}$ \\
\hline $\begin{array}{l}\text { Escala de Resiliencia (BRS) } \\
\text { (Smith et al., 2008) } \\
\text { Escala de Resiliencia parental } \\
\text { frente a los problemas de conduc- } \\
\text { ta de los hijos. (BRS-PC) } \\
\text { (Cantero-García y Alonso-Tapia, } \\
\text { 2018b) }\end{array}$ & $\begin{array}{l}\text { BRS: Incluye seis ítems formulados tanto de forma positiva como negativa. } \\
\text { los ítems se responden en una escala Likert señalando el grado de acuerdo } \\
\text { desde } 1 \text { (totalmente en desacuerdo) a } 5 \text { (totalmente de acuerdo). La fiabilidad } \\
\text { en la muestra original fue }=.83 \text { y de } .81 \text { en la muestra de este estudio. } \\
\text { BRS-PC: Incluye seis ítems formulados tanto de forma positiva como negati- } \\
\text { va. los ítems se responden en una escala Likert señalando el grado de acuerdo } \\
\text { desde } 1 \text { (totalmente en desacuerdo) a } 5 \text { (totalmente de acuerdo). La fiabilidad } \\
\text { se evaluó mediante el } \alpha \text { de Cronbach, obteniendo un valor de } \alpha=.84 \text {, indica- } \\
\text { tivo de buena fiabilidad. }\end{array}$ \\
\hline STAI (Spielberg et al., 1982) & $\begin{array}{l}\text { Evalúa la ansiedad. Instrumento de } 40 \text { ítems. Los ítems se responden en una } \\
\text { escala Likert de } 4 \text { puntos }(0=\text { casi nunca/nada; } 1=\text { algo/a veces; } 2=\text { bastante/a } \\
\text { menudo; } 3=\text { mucho/casi siempre). En muestras de la población española se } \\
\text { han encontrado niveles de consistencia interna que oscilan, tanto para la pun- } \\
\text { tuación total como para cada una de las subescalas, entre } .84 \text { y } .93 \text {. }\end{array}$ \\
\hline BDI-II (Beck et al., 1978) & $\begin{array}{l}\text { Evalúa depresión. Instrumento de } 21 \text { ítems. Cada ítem se valora de } 0 \text { a } 3 \text { pun- } \\
\text { tos en función de la alternativa escogida y, tras sumar directamente la puntua- } \\
\text { ción de cada ítem, se puede obtener una puntuación total que varía de } 0 \text { a } 63 \text {. } \\
\text { La consistencia interna es de } .89 \text {, lo que indica una muy buena consistencia } \\
\text { interna. }\end{array}$ \\
\hline \multicolumn{2}{|c|}{$\begin{array}{l}\text { EVALUACIÓN DE LA PERCEPCIÓN DE LA CALIDAD DEL PROGRAMA } \\
\text { Y DE LA SATISFACCIÓN CON EL MISMO }\end{array}$} \\
\hline $\begin{array}{l}\text { Fichas de seguimiento de las } \\
\text { sesiones } \\
\text { (Cantero- García, 2018) }\end{array}$ & $\begin{array}{l}\text { Evalúan paso a paso la valoración de la implementación del programa por los } \\
\text { padres. Consta de } 12 \text { ítems. Cada ítem se valora de } 0 \text { a } 5 \text { (siendo } 0 \text { muy malo } \\
\text { y } 5 \text { muy bueno). }\end{array}$ \\
\hline $\begin{array}{l}\text { Cuestionario de valoración del } \\
\text { programa } \\
\text { (Cantero-García, 2018) }\end{array}$ & $\begin{array}{l}\text { Evalúan la valoración y satisfacción de los padres con el conjunto del progra- } \\
\text { ma. Consta de } 38 \text { ítems y } 9 \text { preguntas abiertas. Cada ítem se valora en una } \\
\text { escala del } 0 \text { al } 5 \text { (siendo el cero muy malo y el } 5 \text { muy bueno) }\end{array}$ \\
\hline
\end{tabular}


Tabla 2. Ejemplo de ítem sobre el Cuestionario de Conocimientos sobre la gestión del comportamiento de los hijos.

Piense que su hijo/a ante una orden hace el siguiente comentario: "No lo hago, no me da la gana”. ¿Cuál de las siguientes formas de actuar es más adecuada?

a. Vale, yo me encargo de hacerlo. Prefiero no escucharte más, pero que esto no se vuelva a repetir.

b. Eres tonto, he dicho que lo haces y no hay más que hablar, tienes que hacerme caso, yo soy tu padre/madre.

c. Entiendo que no te apetezca hacerlo, pero tienes que hacerlo cuanto antes. Te doy dos minutos.

\section{Procedimiento}

El estudio fue aprobado por el Comité de ética de la universidad del tercer autor. Para la selección de la muestra, se contactó con familias de la Comunidad de Madrid a través de los centros educativos y asociaciones de padres y madres, a los que se entregó una carta de presentación. Una vez presentado el programa se procedió a la difusión de este a través de carteles, trípticos, etc., de tal forma que las familias interesadas comunicaron su interés en participar en el programa a la asociación de padres y madres del centro. Los requisitos para poder participar en el programa fueron 1) tener hijas/os con problemas de conducta con las edades anteriormente descritas, y 2) que los hijos no se encontraran en tratamiento farmacológico para el control de dichos problemas. En la sesión inicial se obtuvo el consentimiento informado de todas las familias participantes, así como, la autorización para la participación de sus hijos.

Una vez formados los grupos de cada uno de los seis centros que conformaban el grupo de intervención, se realizó un acto de inauguración con personal de la Universidad, personal del Centro educativo y las familias participantes. A continuación, se inició la aplicación del programa. Al finalizar la intervención se realizó un acto de clausura con la entrega de diplomas a las familias, agradeciendo su participación y reconociendo su implicación.

\section{Análisis de datos}

En primer lugar, se comprobó la equivalencia pretest del grupo de intervención y el grupo control respecto a las variables demográficas mediante pruebas $t$ para diferencias de medias en variables continuas o dicotómicas como la edad o el sexo y pruebas $\chi^{2}$ para dife- 
rencias de proporciones en variables categóricas con tres o más niveles, como el nivel educativo o el estado civil. Estas pruebas revelaron que no había diferencias significativas entre ambos grupos en cuanto a la edad y sexo de los participantes, su nivel de estudios, su estado civil, el número de hijos, la edad de los hijos o el tener un trabajo remunerado. Sí hubo una diferencia levemente significativa en cuanto al sexo de los hijos $(p=.034)$, indicativo de que en el grupo control había mayor proporción de niños (75\%) que en el grupo de intervención $(46,3 \%)$.

En segundo lugar, se comprobó la equivalencia pretest del grupo de intervención y el grupo control respecto a las variables dependientes mediante pruebas $t$ para diferencias de medias. Los resultados indicaron que no hubo diferencias $(p>.05)$ excepto en el caso de dos componentes del Cuestionario de Clima Familiar Percibido por los Padres (RPCD): motivación $(p=.001)$ y actitud positiva $(p=.03)$. En ambas variables, el grupo control mostró una media superior en la evaluación previa.

Para determinar los cambios en las distintas variables evaluadas mediante el diseño anteriormente descrito se realizó de cada una de ellas un análisis de varianza (ANOVA) de dos factores con medidas repetidas. En cuanto a los datos sobre percepción de la calidad del programa y satisfacción con el mismo, se realizaron análisis de tipo descriptivo. Para todos los análisis se utilizó el programa SPSS v. 22

\section{Resultados}

Evaluación del aprendizaje: cambio en los conocimientos.

La Tabla 3 muestra los resultados del ANOVA, que también se presentan en la Figura 2, así como las medias y las desviaciones tipicas de cada grupo correspondiente a cada uno de los componentes del cuestionario de conocimientos. Tal y como puede observarse, el efecto de la interacción ocasión x condición ha resultado significativo en la dirección esperada. No obstante, el tamaño del efecto $\left(\eta^{2}=.09\right)$ es moderado (IBM Knowledge Center, 2018). 
Tabla 3. ANOVA de los efectos del programa sobre los conocimientos de los padres sobre pautas de gestión del comportamiento y sobre estrategias de autorregulación.

\begin{tabular}{|c|c|c|c|c|c|c|c|}
\hline & uente de variación & S.C. & $G L$ & $M C$ & $F$ & $p$ & $\eta 2$ \\
\hline \multirow{3}{*}{$\begin{array}{l}\text { Intra- } \\
\text { sujetos }\end{array}$} & Ocasión & 20.73 & 1 & 20.73 & 5.94 & .01 & .09 \\
\hline & casión * condición & 20.73 & 1 & 20.73 & 5.94 & .01 & .09 \\
\hline & Error (ocasión) & 205.78 & 59 & 3.49 & & & \\
\hline \multirow{2}{*}{$\begin{array}{c}\text { Inter- } \\
\text { sujetos }\end{array}$} & Condición & 319.09 & 1 & 319.69 & 19.74 & .00 & .25 \\
\hline & Error & 953.37 & 59 & 16.15 & & & \\
\hline \multicolumn{8}{|c|}{ Estadísticos descriptivos } \\
\hline & \multicolumn{3}{|c|}{ Antes } & \multicolumn{4}{|c|}{ Después } \\
\hline & Media & \multicolumn{2}{|c|}{$D T$} & \multicolumn{2}{|c|}{ Media } & \multicolumn{2}{|c|}{$D T$} \\
\hline \multirow{2}{*}{$\begin{array}{c}\text { Control } \\
\text { Experimental }\end{array}$} & 13.75 & \multicolumn{2}{|c|}{4.25} & \multicolumn{2}{|c|}{13.75} & \multicolumn{2}{|c|}{4.25} \\
\hline & 16.32 & \multicolumn{2}{|c|}{3.13} & \multicolumn{2}{|c|}{18.07} & \multicolumn{2}{|c|}{1.40} \\
\hline
\end{tabular}

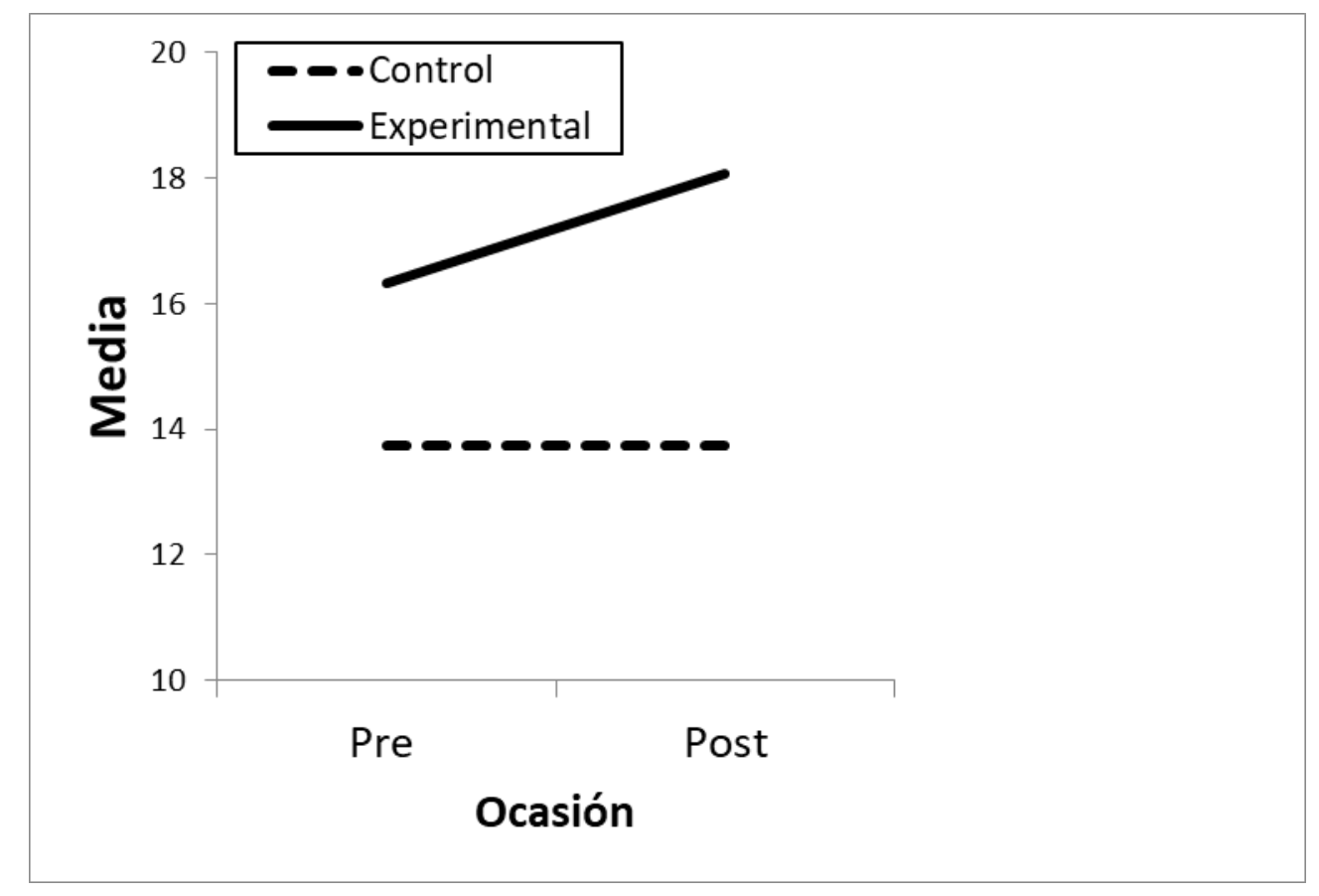

Figura 2. Medias en conocimientos sobre gestión de problemas de conducta 
Evaluación de la transferencia: cambio en el uso de conocimientos.

La Tabla 4 muestra las medias y las desviaciones típicas de cada grupo correspondiente a cada uno de los componentes del Cuestionario de Clima Familiar Percibido por los Padres (RPCD). Los efectos de la interacción ocasión por condición que muestran el cambio relativo entre los grupos experimental y control fueron significativos y en la direccion esperada en las escalas motivación $\left(F / g l 1:=8.456, p=.005 ; \eta^{2}=.12\right)$, control $\left(F / g l 1:=6.927, p=.011 ; \eta^{2}\right.$ $=.10)$, cooperación $\left(F / g l 1:=1.920, p=.17 ; \eta^{2}=.32\right)$, pensamiento positivo $(F / g l 1:=.87$, $\left.p=.004 ; \eta^{2}=.12\right)$ y actitud positiva $\left(F / g l 1:=9.846, p=.003 ; \eta^{2}=.14\right)$, y los tamaños del efecto fueron moderados.

Tabla 4. Evaluación de la Transferencia; RPCD perspectiva de los padres. Medias y desviaciones típicas

\begin{tabular}{lccccc}
\hline & & \multicolumn{3}{c}{ Pre } & \multicolumn{2}{c}{ Post } \\
\cline { 3 - 6 } \multicolumn{1}{c}{ Variables } & $\mathrm{G}^{1}$ & Media & DT & Media & $D T$ \\
\hline \multirow{2}{*}{ Motivación } & $\mathrm{C}$ & 4.18 & .67 & 4.16 & .70 \\
& $\mathrm{E}$ & 3.43 & 1 & 4.13 & .73 \\
Control & $\mathrm{C}$ & 2.88 & 1 & 2.68 & .96 \\
& $\mathrm{E}$ & 2.47 & .89 & 3.03 & .83 \\
Cooperación & $\mathrm{C}$ & 2.50 & .36 & 3.71 & .91 \\
& $\mathrm{E}$ & 2.57 & .62 & 4.08 & .53 \\
Pensamiento & $\mathrm{C}$ & 3.96 & .55 & 3.95 & .57 \\
Positivo & $\mathrm{E}$ & 3.78 & .77 & 4.44 & .50 \\
Actitud Positiva & $\mathrm{C}$ & 4.13 & .62 & 4.11 & .48 \\
& $\mathrm{E}$ & 3.72 & .66 & 4.26 & .46
\end{tabular}

${ }^{1} \mathrm{G}$ : group; C: Control; E: Experimental.

La Tabla 5 muestra las medias y las desviaciones tipicas de cada grupo correspondientes al Cuestionario de Estrategias de Afrontamiento (CAF-PC), así como, los índices correspondientes a los componentes del CAF-PC. La Tabla 5 recoge los valores $F$ y los parciales de $\eta^{2}$ correspondientes al efecto de la ocasión x condición. Como se puede apreciar sólo las estrategias de afrontamiento: autoculpabilización, relajación, esperar y pensamiento positivo han mostrado cambios significativos en la dirección esperada en el grupo experimental. 
Tabla 5. Evaluación de la Transferencia; CAF-PC. Medias y desviaciones típicas

\begin{tabular}{|c|c|c|c|c|c|}
\hline \multirow[b]{2}{*}{ Variables } & \multirow[b]{2}{*}{$\mathrm{G}^{1}$} & \multicolumn{2}{|c|}{ Pre } & \multicolumn{2}{|c|}{ Post } \\
\hline & & Media & $D T$ & Media & $D T$ \\
\hline \multirow{2}{*}{ Autoculpabilización } & $\mathrm{C}$ & 3.05 & .57 & 3.16 & .46 \\
\hline & $\mathrm{E}$ & 3.21 & .68 & 2.57 & .73 \\
\hline \multirow{2}{*}{ Impulsividad } & $\mathrm{C}$ & 3.18 & .46 & 3.11 & .47 \\
\hline & $\mathrm{E}$ & 3.21 & .49 & 2.94 & .42 \\
\hline \multirow{2}{*}{ Rumiación } & $\mathrm{C}$ & 2.98 & .66 & 2.95 & .74 \\
\hline & $\mathrm{E}$ & 3.26 & .67 & 3.47 & .44 \\
\hline \multirow{2}{*}{ Aislamiento } & $\mathrm{C}$ & 2.41 & .55 & 2.43 & .58 \\
\hline & $\mathrm{E}$ & 2.67 & .66 & 2.57 & .68 \\
\hline \multirow{2}{*}{ Relajación } & $\mathrm{C}$ & 3.05 & .69 & 2.93 & .68 \\
\hline & $\mathrm{E}$ & 2.93 & .73 & 3.50 & .91 \\
\hline \multirow{2}{*}{ Esperar } & $\mathrm{C}$ & 2.81 & .75 & 2.75 & .77 \\
\hline & $\mathrm{E}$ & 2.74 & .79 & 3.38 & 1 \\
\hline \multirow{2}{*}{ Pensamiento Alegre } & $\mathrm{C}$ & 3.15 & .48 & 3.11 & .52 \\
\hline & $\mathrm{E}$ & 3.15 & .62 & 2.91 & .51 \\
\hline \multirow{2}{*}{ Evitar Pensar } & $\mathrm{C}$ & 3.06 & .73 & 3.03 & .68 \\
\hline & $\mathrm{E}$ & 3.21 & .68 & 3.17 & .52 \\
\hline \multirow{2}{*}{ Reintentar } & $\mathrm{C}$ & 3.20 & .65 & 3.23 & .57 \\
\hline & $\mathrm{E}$ & 3.01 & .79 & 3.52 & .58 \\
\hline \multirow{2}{*}{ Solucionar Problemas } & $\mathrm{C}$ & 2.81 & .38 & 2.80 & .39 \\
\hline & $\mathrm{E}$ & 2.95 & .43 & 2.75 & .36 \\
\hline \multirow{2}{*}{ Búsqueda de Ayuda } & $\mathrm{C}$ & 2.43 & .65 & 2.50 & .79 \\
\hline & $\mathrm{E}$ & 2.47 & .62 & 2.50 & .48 \\
\hline \multirow{2}{*}{ Pensamiento Positivo } & $\mathrm{C}$ & 3.18 & .41 & 3.21 & .46 \\
\hline & $\mathrm{E}$ & 3.30 & .60 & 2.83 & .63 \\
\hline \multirow{2}{*}{$\begin{array}{l}\text { Autorregulación } \mathrm{Ne}- \\
\text { gativa de las Emocio- } \\
\text { nes }\end{array}$} & $\mathrm{C}$ & 2.90 & .86 & 2.91 & .77 \\
\hline & $\mathrm{E}$ & 3.09 & .60 & 2.89 & .54 \\
\hline \multirow{2}{*}{$\begin{array}{l}\text { Autorregulación Posi- } \\
\text { tiva de las Emociones }\end{array}$} & $\mathrm{C}$ & 3.02 & .10 & 2.95 & .11 \\
\hline & $\mathrm{E}$ & 3.01 & .07 & 3.24 & .08 \\
\hline \multirow{2}{*}{$\begin{array}{l}\text { Afrontamiento Cen- } \\
\text { trado en el Problema }\end{array}$} & $\mathrm{C}$ & 2.90 & .08 & 2.93 & .09 \\
\hline & $\mathrm{E}$ & 2.93 & .06 & 2.90 & .06 \\
\hline
\end{tabular}

${ }^{1}$ G: grupo. C: Control. E: Experimental. 


\section{Evaluación del Impacto}

Impacto percibido por los hijos.

La tabla 6 recoge las medias y las desviaciones típicas de cada grupo correspondientes al Cuestionario de Clima familiar Percibido por los Hijos (CFPH). Los efectos de la interacción ocasión por condición que muestran el cambio relativo entre los grupos experimental y control fueron significativos y en la direccion esperada en las escalas paciencia $(F / g l 1:=$ $\left.1.155, p=.028 ; \eta^{2}=.019\right)$, chillidos $\left(F / g l 1:=.518, p=.047 ; \eta^{2}=.009\right)$ y razonamiento $\left(F / g l 1:=1.365, p=.024 ; \eta^{2}=.023\right)$ pero el tamaño del efecto no es significativo.

Tabla 6. Impacto: Medias y desviaciones típicas en el Clima familiar percibido por los hijos.

\begin{tabular}{lccccc}
\hline & & \multicolumn{2}{c}{ Pre } & \multicolumn{2}{c}{ Post } \\
\cline { 3 - 6 } Variables & $\mathrm{G}^{1}$ & Media & DT & Media & DT \\
\hline \multirow{2}{*}{ Paciencia } & $\mathrm{C}$ & 4.06 & .58 & 4.42 & .62 \\
& $\mathrm{E}$ & 4.29 & .57 & 4.41 & .60 \\
Actitud Positiva & $\mathrm{C}$ & 3.91 & .46 & 4.16 & .50 \\
& $\mathrm{E}$ & 3.85 & .74 & 4.21 & .65 \\
Chillidos & $\mathrm{C}$ & 3.13 & .79 & 2.93 & .87 \\
& $\mathrm{E}$ & 2.97 & .89 & 2.58 & .99 \\
Razonamiento & $\mathrm{C}$ & 3.95 & .52 & 4.24 & .64 \\
& $\mathrm{E}$ & 4.20 & .61 & 4.24 & .64 \\
\multirow{2}{*}{ Satisfacción } & $\mathrm{C}$ & 3.80 & .93 & 4.11 & .83 \\
& $\mathrm{E}$ & 4.22 & .73 & 4.46 & .59 \\
\hline
\end{tabular}

${ }^{1}$ G: grupo. C: Control. E: Experimental.

\section{Impacto percibido por los padres.}

Escala breve de resiliencia (BRS). Los participantes del grupo experimental han cambiado en mayor medida la percepción sobre la resiliencia, el cambio se ha producido en la dirección esperada, sin embargo, el tamaño del efecto no es significativo $(F / d f 1:=2.301, p=$ $\left..135 ; \eta^{2}=.038\right)$

Cuestionario de Resiliencia Parental frente a los Problemas de conducta de los hijos (BRS-PC). Los participantes del grupo experimental han cambiado en mayor medida la per- 
cepción sobre la resiliencia frente a los problemas de conducta de los hijos $(F / d f 1:=1.268, p$ $\left.=.265 ; \eta^{2}=.021\right)$ y el cambio se ha producido en la dirección esperada; sin embargo, el tamaño del efecto no es significativo.

Cuestionario de Estrés parental. El efecto de la interacción ocasión x condición no ha sido significativo $(p>.05)$. Los participantes del grupo experimental no han cambiado en mayor medida la percepción sobre el estrés parental.

Inventario de Ansiedad (STAI). La figura 3 recoge las medias de cada grupo. El efecto de la interacción ocasión x condición es significativo y en la dirección esperada $(F / g l 1:=$ 8.202, $p=.006 ; \eta^{2}=.12$ ).

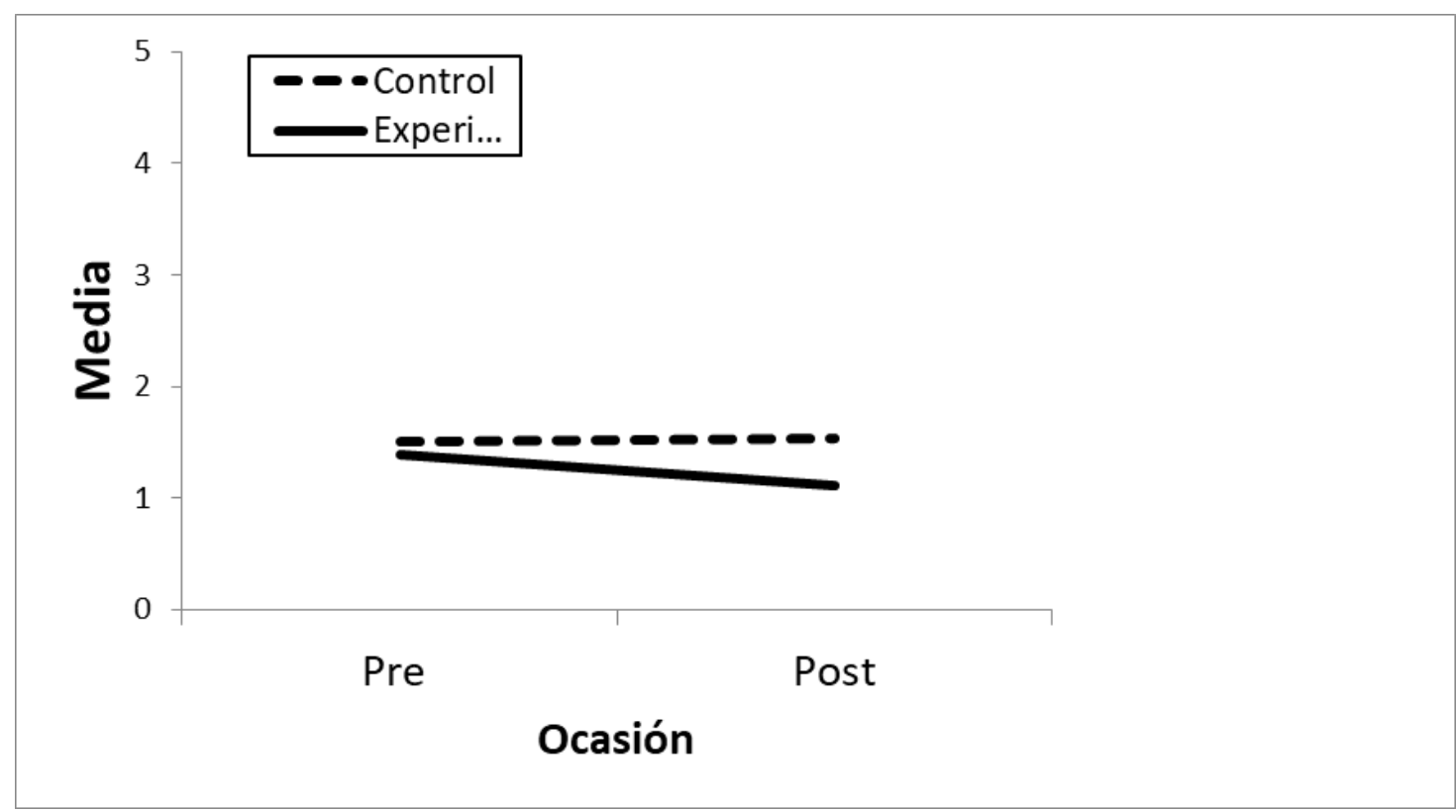

Figura 3. Medias en los niveles de ansiedad: Cuestionario STAI-Rasgo.

Inventario de Depresión (BDI). La figura 4 recoge las medias de cada grupo. El efecto de la interacción ocasión x condición ha sido significativo y en la dirección esperada $(F / d f 1$ : $=14.143, p<.001)$ con un tamaño del efecto moderado $\left(\eta^{2}=.19\right)$. 


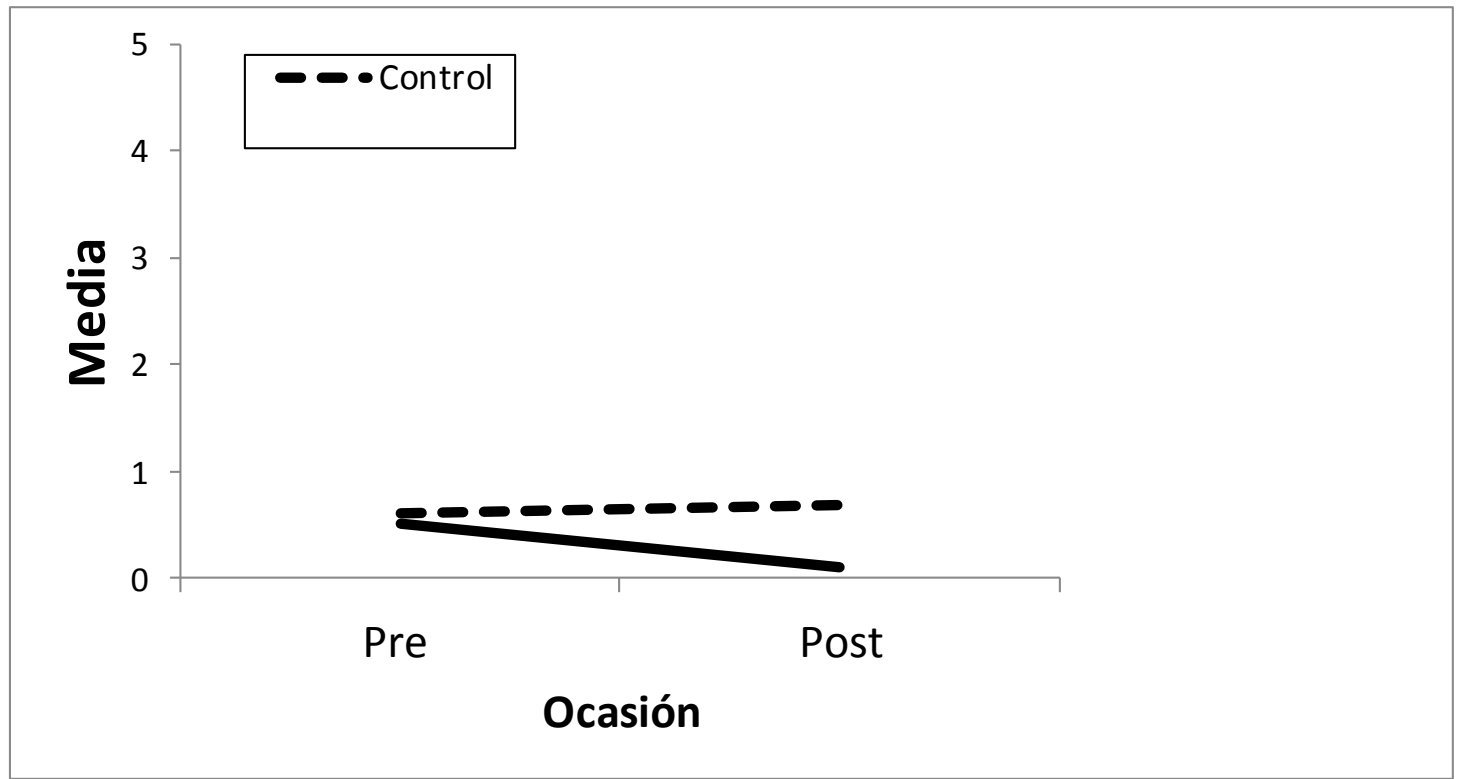

Figura 4. Media en los niveles de depresión: Cuestionario BDI

Evaluación de la percepción de la calidad del programa.

Las puntuaciones de satisfacción se han agrupado en tres grandes apartados: 1) satisfacción general con la calidad del programa y lo aprendido, 2) satisfacción con la forma de enseñar 3) y satisfacción con las técnicas utilizadas. En todos los casos las variables se evalúan en una escala de cinco puntos, desde 1 (muy malo) a 5 (muy bueno).

En cuanto a la satisfacción general con el programa, así como con las cuatro facetas, es muy buena cómo muestra la Tabla 7. Lo mismo ocurre con la forma de enseñar (Tabla 7). $\mathrm{Y}$ en cuanto a la satisfacción con la calidad de las técnicas enseñadas, la valoración ha sido muy positiva: 1$)$ elogio $(M=4.84, D T=.37) ; 2)$ técnica del sándwich $(M=4.55, D T=.55)$; 3) el uso de los mensajes "yo" $(M=4.50, D T=.55)$; 4) la técnica de ignorar los comportamientos poco importantes $(M=4.66, D T=.58) ; 5)$ el uso de los premios $(M=4.39, D T=$ $.63), 6)$ el establecer normas y límites $(M=4.71, D T=.61)$; 7) la técnica de los cinco minutos de queja $(M=4.45, D T=.68) ; 8)$ la técnica de resolución de conflictos "yo gano-tú ganas" $(M=4.47, D T=.64) ; 9)$ el momento especial con mis hijos $(M=4.74, D T=.55) ; 10)$ los ejercicios para trabajar las distintas estrategias de afrontamiento $(M=4.61, D T=.49)$; 11) las meditaciones $(M=4.42, D T=.68) ; 12)$ las reflexiones con el resto del grupo $(M=4.66, D T=$ $.63)$; y 13) los ejercicios que se han trabajado para mejorar mi bienestar $(M=4.79, D T=.47)$. 
Tabla 7. Satisfacción: Medias y desviaciones típicas en la satisfacción con el programa y con la forma de enseñar

\begin{tabular}{lcc}
\hline \multicolumn{1}{c}{ Variables } & Media & DT \\
\hline Satisfacción general con el programa & 4.73 & .19 \\
Satisfacción con la reducción de los problemas de conducta del hijo & 4.45 & .53 \\
Satisfacción personal de los padres & 4.60 & .40 \\
Capacidad para manejar los problemas de conducta del hijo & 4.29 & .51 \\
Recomendación del programa & 4.92 & .27 \\
Satisfacción con la forma de enseñar & 4.63 & .36 \\
\hline
\end{tabular}

\section{Discusión y Conclusiones}

El objetivo del presente estudio fue comprobar si la aplicación del programa de entrenamiento ¡Supérate: no tires la toalla!, diseñado para promover la mejora de las estrategias de afrontamiento del estrés y las estrategias de manejo de los problemas de conducta de los hijos y, con ello, el sentido de autoeficacia, facilitaba el aumento de la resiliencia de los padres, así como la disminución de los niveles de ansiedad y depresión.

Para conseguir el objetivo señalado, el programa desarrollado incorpora estrategias de manejo de los problemas de comportamiento que habitualmente se incluyen en los programas de entrenamiento de padres con hijos con problemas de conducta (Kaminskiet al., 2008) pero, además, entrena estrategias específicas de afrontamiento y autorregulación de las emociones. Otra de las aportaciones del programa es la incorporación de distintas técnicas y tareas para garantizar la aplicación de las pautas trabajadas en las distintas sesiones, para lo cual se emplearon autorregistros y retroalimentación sobre las habilidades enseñadas. Estos métodos que aquí se señalan son poco trabajados habitualmente en los programas de entrenamiento, tal y como ponen de manifiesto algunos estudios de revisión (Lozano y Valero, 2017; VivesMontero, Cortés-Pendón, López-Rubio y Ascanio, 2017).

Las hipótesis planteadas para este estudio tienen que ver en primer lugar con el aprendizaje de los conocimientos impartidos en el programa, en segundo lugar, con la transferencia 
de estos, en tercer lugar, con su impacto y, finalmente, con la percepción de la calidad del programa y con la satisfacción con el mismo.

En primer lugar, las hipótesis relacionadas con aprendizaje sugerían que los conocimientos de los padres sobre las estrategias a utilizar para el manejo de los problemas de comportamiento de los hijos, y los conocimientos sobre cómo manejar el estrés mejorarían de manera significativa. Los resultados apoyan esta hipótesis. Tal y como indican los resultados de este estudio, estos conocimientos mejoran tras la aplicación del programa de entrenamiento, puesto que los conocimientos del grupo experimental sobre las pautas de actuación para la mejora de los problemas de comportamiento de los hijos, así como para el manejo de su estrés fueron significativamente superiores a los del grupo de control. Los resultados obtenidos en este estudio son similares a los que se han encontrado en estudios previos (Bloomfield y Kendall, 2012; Borrego y Burrell, 2010, Sanders, Baker y Tuner, 2012; Vives et al., 2017; Wilson, Havighurst y Harley, 2012).

En segundo lugar, con respecto a las hipótesis relacionadas con la transferencia, se esperaba que los padres mejoraran el uso de las estrategias de afrontamiento y autorregulación emocional, como también su percepción del clima familiar. Los resultados muestran que, de todas las estrategias de afrontamiento trabajadas en el programa, solo autoculpabilización, relajación, esperar y pensamiento positivo han mostrado cambios significativos. No obstante, pese a que las estrategias de afrontamiento que los padres utilizan de cara a los problemas de conducta de sus hijos mejoran, los niveles de resiliencia no lo han hecho. Este resultado puede deberse a la duración de intervención, ya que es posible que diez sesiones no sean suficientes para abordar de manera completa las distintas estrategias de afrontamiento.

En relación, con el clima familiar percibido por los padres, los resultados ponen de manifiesto que los cambios son significativos en cuanto a la motivación, el control y el pensamiento positivo en relación con los problemas de conducta de sus hijos. Estos resultados van en línea con los estudios previos (Martínez-González, Rodríguez-Ruiz, Álvarez-Blanco, Becedóniz-Vázquez, 2016; Ponzetti, 2016; Robles y Romero, 2011, Rodrigo, Máiquez y Martín, 2015) en los que se constatan la percepción de control, el incremento de la motivación, pensamiento positivo, autoestima y asertividad tras recibir la formación parental. 
En tercer lugar, se esperaba que el programa tuviera impacto positivo y significativo en: a) disminución del estrés parental, los niveles de ansiedad y depresión b) el clima familiar percibido por los hijos. Cabe señalar que solo estrés parental, ansiedad y depresión, experimentaron dicha disminución. En el resto de las variables no se han producido cambios. Estos resultados pueden estar relacionados con el tamaño muestral, la duración de las sesiones y con la duración del programa en general, así como que dentro de la familia se presente la situación idónea para afrontarla de manera competente. Líneas futuras de investigación deberían tener en cuenta estos aspectos e incluir más sesiones o ampliar la duración de las mismas para trabajar aspectos que influyan en dichas variables, así como el modo especifico de trabajarlas.

En cuarto lugar, cabía esperar que la percepción de los padres sobre los problemas de conducta de los hijos en el ámbito familiar mejorara tras la aplicación del programa, no obstante, los cambios que se detectaron no fueron significativos. Esto puede ser debido al momento en el que se ha realizado la evaluación pos-test. Consideramos que sería necesario realizar un seguimiento en los próximos meses para ver si estos resultados se han consolidado.

Por último, cabía esperar un elevado grado de satisfacción con el programa en su conjunto y con las distintas características del programa en particular. Los resultados han puesto de manifiesto que la satisfacción del grupo experimental es elevada. Además, este alto grado de satisfacción con el programa, junto con el hecho de que muchos de los cambios a los que se hacía referencia en las tres primeras hipótesis no hayan sido significativos, sugiere que una mayor continuidad sería adecuada. La metodología seguida, la aplicación y sistematicidad de las sesiones ha contribuido a estos resultados. Estos datos van en línea con lo que recogen estudios como el de Dretzke et al., (2005), Lundhal, Risser y Lovejoy (2006), McCart, Priester, Davies y Azen (2006) en los que se indica que los programas de educación parental, que siguen esta metodología son una de las intervenciones más eficaces para trabajar las competencias parentales de padres y madres con preadolescentes y adolescentes. Por lo tanto, podemos señalar que la valoración del proceso de implementación del programa ;Supérate: no tires la toalla! ha mostrado: 1) que este es viable y se ajusta a los criterios y recomendaciones que se proponen en la literatura científica; 2) que permite mejorar algunas de las prácticas educativas de los padres ante los problemas de conducta del niño 2) y muchas de las estrategias de afrontamiento y autorregulación de los padres; 3) que su metodología, contenidos, materiales de trabajo y técnicas de entrenamiento son aceptadas y percibidas como útiles por los participantes; 4) que genera una baja tasa de abandono y una elevada asistencia e implica- 
ción y 5) que genera una alta satisfacción entre los participantes. En general, pues, se puede concluir que el programa se presenta como una herramienta eficaz en la intervención de los niveles de algunas estrategias de afrontamiento que van a influir en la resiliencia de los padres y en los problemas de conducta de los hijos en la preadolescencia y adolescencia.

No obstante, este estudio tiene ciertas limitaciones que merecen atención. En primer lugar, el número de madres que participan en el programa de entrenamiento es bastante mayor que el número de padres, por lo que se ha podido generar sesgo en cuanto al género. Además, cabe destacar que un $70 \%$ de las y los participantes contaban con estudios universitarios, lo que indica que la muestra está sesgada hacia un nivel educativo alto y ha podido influir de alguna forma en los resultados, por lo que deben generalizarse con suma cautela. Adicionalmente, casi el $90 \%$ de las familias que participan en el programa de intervención estaban constituidas por progenitores casados/as. En este sentido sería necesario explorar en futuras investigaciones si existen diferencias en los resultados al aplicar estos programas en los distintos niveles educativos y tipos de familias (e.g., familias monoparentales o de progenitores separados o divorciados). También es necesario llamar la atención sobre el hecho de que, si bien el grupo de intervención estaba conformado de manera equilibrada por dos centros de cada tipo (públicos, privados y concertados), el grupo control estuvo formado por los participantes provenientes de un centro público, con mayor proporción de hijos varones y una motivación y actitud positiva iniciales superiores. Estas cuestiones pueden haber tenido implicaciones a la hora de generar los contrastes de resultados. Recomendamos que las futuras investigaciones obtengan una muestra más amplia que permita un grupo control más equivalente al experimental.

Una fortaleza importante de este estudio estudio es que presenta un completo sistema de evaluación del programa descrito, basado en instrumentos previamente validados. Dicha propuesta de evaluación podría constituirse como sistema de evaluación basado en evidencias, inicialmente utilizado en este estudio piloto con una muestra más reducida. En el futuro, convendría que las sucesivas aplicaciones del programa hicieran uso del mismo para estandarizar la identificación de la capacidad de cambio que dicho programa promueve. 


\section{Referencias}

Abidin, R.R. (1995). Parenting Stress Index (PSI) manual (3 ${ }^{\mathrm{a}}$ ed.). Charlottesville, VA: Pediatric Psychology Press.

Alonso-Tapia, J. (2012). Evaluación psicopedagógica y orientación educativa. Madrid: Síntesis.

Alonso-Tapia, J., Garrido-Hernansaiz, H., Rodríguez-Rey, R., Ruiz, M., \& Nieto, C. (2017). Personal factors underlying resilience: development and validation of the Resiliency Questionnaire for Adults. International Journal of Mental Health Promotion, 19(2), 104-117. http://dx.doi.org/10.1080/14623730.2017.1297248

Atienza, F. L., Pons, D., Balaguer, I., y García Merita, M. (2000). Propiedades psicométricas de la Escala de Satisfacción con la Vida en adolescentes. Psicothema, 12(2), 314-319.

Azar, S. T., Cote, L. R. (2002). Sociocultural issues in the evaluation of the needs of children in custody decision making: What do our current frameworks for evaluating parenting practices have to offer? International Journal of Law and Psychiatry, 25(3), 193-217.

Barkley, R. A., Murphy, K. R. y Bauermeister, J. J. (1997). Niños desafiantes: Materiales de evaluación y folletos para los padres. Reimpreso en "Trastorno por déficit de atención e hiperactividad: un manual de trabajo clínico”. Nueva York: The Guilford Press.

Beck, A. T., Steer, R. A., y Brown, G. K. (1996). Beck depression inventory-II. San Antonio, 78(2), 490-8.

Bloomfield, L y Kendall,S. (2012). Parenting self- efficacy, parenting stress and child behavior before and after a parenting program. Primary Health Care Research y Develo ment, 13, 364-372. https://doi.org/10.1017/S1463423612000060

Borrego, J. y Burrell, T. L. (2010). Using behavioral parent training to treat disruptive behavior disorders in young children. Cognitive and Behavioral Practice, 17, 25-34. http://dx.doi.org/10.1016/j.cbpra.2009.10.003

Cantero- García, M., y Alonso-Tapia, J (2018a) Estrategias de afrontamiento y resiliencia en familias con hijos con problemas de conducta. Revista de Psicodidáctica, 23(2), 153 159. https://doi.org/10.1016/j.psicod.2018.04.001

Cantero-García, M. (2018). Resiliencia frente a los problemas de conducta de los hijos. Tesis doctoral no publicada. Universidad Autónoma de Madrid.

Cantero-García, M., y Alonso-Tapia, J. (2017a). Cuestionario breve de respuesta parental ante el comportamiento disruptivo (RPCD): Perspectiva de los padres. Anales de psicología, 33(3), 689-696. http://dx.doi.org/10.6018/analesps.33.3.266971 
Cantero-García, M., y Alonso-Tapia, J. (2017b). Evaluación del clima familiar creado por la gestión de los problemas de conducta, desde la perspectiva de los hijos. Electronic Journal of Research in Educational Psychology, 15(2), 259-280. http://dx.doi.org/10.14204/ejrep.42.16062

Cantero-García, M., y Alonso-Tapia, J. (2018). Escala Breve de Resiliencia frente a los Problemas de Comportamiento de los hijos (EBR-PC). Anales de Psicología/Annals of Psychology, 34(3), 531-535. http://dx.doi.org/10.6018/analesps.34.3.312601

Cantero-García, M., y Alonso-Tapia, J. (2018c). Cuestionario de Conocimientos previos sobre gestión del comportamiento y afrontamiento del estrés. Trabajo no publicado. Universidad Autónoma de Madrid.

Chainé, S. M., Ruíz, M. J. M., del Campo Sánchez, R. M., y Gutiérrez, J. N. (2016). Las prácticas de crianza y la reducción de los problemas de conducta infantil. Psicología Conductual, 24(2), 341.

Chorpita, B. F., Daleiden, E. L., Ebesutani, C., Young, J., Becker, K. D., Nakamura, B. J., ... y Smith, R. L. (2011). Evidence-based treatments for children and adolescents: An updated review of indicators of efficacy and effectiveness. Clinical Psychology: Science and Practice, 18(2), 154-172.

Consejo de Europa (2006). Recomendación Rec (2006)19 del Comité de Ministros a los Estados Miembros sobre políticas de apoyo al ejercicio positivo de la parentalidad.

Dretzke, J., Frew, E., Davenport, C., Barlow, J., Stewart-Brown, S., Sandercock, J., et al. (2005). The effectiveness and cost-effectiveness of parent training/education programs for the treatment of conduct disorder, including oppositional defiant disorder, in children. Health Technology Assessment, 9, 1-233.

Flay, B., Biglan, A., Boruch, R. F., González, F., Gottfredson, D., Kellam, S. ... Ji, P. (2005). Standards of evidence: Criteria for efficacy, effectiveness and dissemination. Prevention Science, 6(3), 151-175.

Forehand R, Jones DJ, Parent J. (2013). Behavioral Parenting Interventions for Child Disruptive Behaviors and Anxiety: What's different and what's the same? Clinical Psychology Review. 33,133-145. https://doi.org/10.1016/j.cpr.2012.10.010

Galbraith, D. D. \& Fouch, S. E (2007). Principles of Adult Learning: Application to Safety Training. Professional Safety, 52(9), 35-40. Recuperado de http://0search.proquest.com.millenium.itesm.mx/docview/200415451?accountid=41938

Gottfredson, D. C., Cook, T. D., Gardner, F. E., Gorman-Smith, D., Howe, G.W., Sandler, I. N., y Zafft, K. M. (2015). Standards of evidence for efficacy, effectiveness, and scale- 
up research in prevention science: Next generation. Prevention Science,16(7), 893926.

Grant, S., Mayo-Wilson, E., Montgomery, P., Macdonald, G., Michie, S., Hopewell, S., \& Moher, D. (2018). CONSORT-SPI 2018 Explanation and Elaboration: guidance for reporting social and psychological intervention trials. Trials, 19(1), 406.

Herreros, O., Sánchez, F., Rubio, B., y Gracia, R. (2004). Actualización en el tratamiento farmacológico de los trastornos del comportamiento de la adolescencia. Monografías de Psiquiatría: Los trastornos de comportamiento en la adolescencia, 1, 60-69. http://dx.doi.org/10.1080/15374416.2013.804386

IBM Knowledge Center (2018). Watson analytics. Términos estadísticos: Eta cuadrado. https://www.ibm.com/support/knowledgecenter/es/SS4QC9/com.ibm.solutions.wa _an_overview.2.0.0.doc/etasquared.html

Johnston, C., y Mash, E. J. (1989). A measure of parenting satisfaction and efficacy. Journal of Clinical and Child Psychology, 18(2), 167-175.

Kaminski, J. W., Valle, L. A., Filene, J. H., y Boyle, C. L. (2008). A meta-analytic review of components associated with parent training program effectiveness. Journal of abnormal child psychology, 36(4), 567-589.

Lochman J.E., y Wells, K.C (2002). The coping power program at the middle school transition: Universal and indicated prevention effects. Psychology of Addictive Behaviors, 16, S40-S54.

Lochman, J. E., Wells, K. C., Murray, M., Tolan, P., Szapocznik, J., y Sambrano, S. (2007). The Coping Power Program: Preventive intervention at the middle school transition. Preventing youth substance abuse: Science-based programs for children and adolescents, 185-210.

Lozano-Rodríguez, I., y Valero-Aguayo, L. (2017). Una revisión sistemática de la eficacia de los programas de entrenamiento a padres. Revista de Psicología Clínica con Niños y Adolescentes, 4(2), 85-91.

Lundhal, B., Risser, H.J., y Lovejoy, M.C. (2006). A meta-analysis of parent training: Moderators and follow - up effects. Clinical Psychology Review, 26, 86-104. https://doi.org/10.1016/j.cpr.2005.07.004 
Martinez-Gónzalez, R.A., Rodríguez-Ruíz, B, Álvarez-Blanco, L., y Becedóniz-Vázquez, C. (2016). Evidencias del fomento de la parentalidad positiva mediante el Programa-Guía para el desarrollo de competencias emocionales, educativas y parentales. Psychosocial Intervention 25 (2), 2 111-117.http://dx.doi.org/10.1016/j.psi.2016.04.001

McCart, M. R., Priester, P. E., Davies, W. H., \& Azen, R. (2006). Differential effectiveness of behavior parent-training and cognitive behavioral therapy for antisocial youth: A meta-analysis. Journal of Abnormal Child Psychology, 34, 527-541.

Oliva, A., Hidalgo, M.V., Martín, D., Parra, A., Ríos, M., Vallejo, R. (2007). Programa de apoyo a madres y padres de adolescentes. Sevilla: Consejería de Salud de la Junta de Andalucía.

Orte, C., Ballester, L., y March, M.X. (2013). El enfoque de la competencia familiar. Una experiencia de trabajo socioeducativo con familias. Pedagogía Social. Revista Interuniversitaria, 21, 13-37. http://www.redalyc.org/articulo.oa?id=135029517002

Orte, C., Touza, C., Ballester, LL, y March, M.X. (2008). Children of drug-dependent parents: prevention programme outcomes. Educational Research, 50(3), 249-260. https://doi.org/10.1080/00131880802309390

Pardo, A., y San Martín, R. (2001). Niveles de indagación: descriptivo, relacional y explicativo. Análisis de Datos en Psicología II. Madrid, Síntesis.

Ponzetti, J.J. (2016). Evidence-based parenting education: A global perspective. London: Routledge.

Robles, Z., y Romero, E. (2011). Programas de entrenamiento para padres de niños con problemas de conducta: una revisión de su eficacia. Anales de psicología, 27, 86-101.

Rodrigo, M. J., Máiquez, M. L., Martín, J. C., Byrne, S., y Rodríguez, B. (Eds.). (2015). Manual práctico de parentalidad positiva (Practical handbook on positive parenting). Madrid: Síntesis.

Rodríguez, E, Martín J.C., y Rodrigo, M.J (2015). "Vivir la Adolescencia en Familia": Un programa de apoyo parental en la adolescencia. En M. J, Rodrigo (Eds.) Manual práctico de parentalidad positiva. (pp. 131-149) Madrid: Síntesis.

Romero, E., Villar, P., Luengo, M.A., Gómez-Fraguela, J.A. y Robles, Z. (2005). Empecemos: Emociones, Pensamientos y Conductas para un desarrollo saludable. Programa para padres. Santiago: Tórculo.

Sanders, M.R., Markie-Dadds, C., y Turner, K.M.T. (2003). Theoretical, scientific and clinical foundations of the Triple P- positive parenting program: A population approach to 
the promotion of parenting competence. Parenting Research and Practice Monograph, $1,1-24$.

Smith, B. W., Dalen, J., Wiggins, K., Tooley, E., Christopher, P., y Bernard, J. (2008). The brief resilience scale: assessing the ability to bounce back. International journal of behavioral medicine, 15(3), 194-200.

Speilberger, C. D., y Vagg, P. R. (1984). Psychometric properties of the STAI: a reply to Ramanaiah, Franzen, and Schill. Journal of personality assessment, 48(1), 95-97.

Vives-Montero, C., Cortés-Pendón, D., López-Rubio, S., y Ascanio, L. (2017). Efectos de un programa de formación de padres para reducir conductas perturbadoras infantiles. Revista de Psicopatología y Psicología Clínica,22(3), 219-227. https://doi.org/10.5944/rppc.vol.22.num.3.2017.18299

Wilson, K. R., Havighurst, S.S., y Harley. A. E. (2012). Tuning in to kids: An effectiveness trial of a parenting program targeting emotion socialization of preschoolers. Journal of family Psychology, 26, 56-65. https://doi.org/10.1037/a0026480 